Research Article

\title{
Structural Improvement of the w-Type High-Speed Rail Clip Based on a Study of Its Failure Mechanism
}

\author{
Xiaogang Gao $(\mathbb{D}$, Anbin Wang, Yu He, and Xiaohan Gu \\ School of Urban Rail Transportation, Shanghai University of Engineering Science, Shanghai 201620, China \\ Correspondence should be addressed to Xiaogang Gao; xgg728@163.com
}

Received 17 April 2019; Revised 17 June 2019; Accepted 25 June 2019; Published 21 July 2019

Academic Editor: Jean-Jacques Sinou

Copyright ( 2019 Xiaogang Gao et al. This is an open access article distributed under the Creative Commons Attribution License, which permits unrestricted use, distribution, and reproduction in any medium, provided the original work is properly cited.

In the circumstances of high-speed railways, the wheel-rail vibration is significantly aggravated by polygonal wheel wear and rail corrugation, which subsequently leads to the wheel-rail interaction at higher frequencies and potential failure of the rail fastening. In this paper, a $\omega$-type clip of the fastening in the $\mathrm{CRH}$ high-speed rail was used to investigate the failure mechanism. First, a dynamic wheel-rail coupling model and a finite element analysis of the rail clip were developed, from which the rail vibration frequency and modal frequencies of the clip with different installation torques were obtained. The experimental tests and modal simulation results were mutually verified. In addition, the real-time vibration measurement and the wheel-rail wear monitoring were carried out at a CRH high-speed railway site. It was found that the resonant frequencies of the $\omega$-type clip in the installation condition coincided with the excitation frequencies of the wheel-rail interaction induced by wheel-rail wear. The high-frequency dynamic failure mechanism of a typical $\omega$-type clip, W300-1, is put forward for the first time. Moreover, a high-frequency rail clip fatigue test system was designed and developed specifically for this study. The loading excitation frequency of the clip test used was set as $590 \mathrm{~Hz}$, and the loading amplitude was $0.05 \mathrm{~mm}$. After 125-minute operation of the test system, the clip was broken at the expected location predicted by the FEA model. The high-frequency fatigue test result further verified that the failure mechanism of the $\omega$-type clip was due to the resonance of the clip with its excitation force from the wheel-rail interaction. Finally, the clip was then structurally improved taking into account the stiffness and mass, which led to its resonant frequencies shifting away from the high-frequency excitation range, hence avoiding resonance failure of the subject clip.

\section{Introduction}

A rail fastening system is a key component for the track system in high-speed railways. Sadeghi and Barati studied the railway design approaches including fastenings proposed by various standards along with the results of a wide range of technical research studies and made necessary suggestions for the improvement of current practices in the analysis and design of the railway tracks [1]. For a fastening system, besides its traditional functions, it also provides vibration insulation which, in a ballasted track system, can be subsidized by ballasts under rail sleepers. In a common $\omega$-type clip fastening system, clips are mounted on top of the gauge block and insulation pad with bolts, as illustrated in Figure 1. The $\omega$-type fastening clips are widely used in high-speed railways.
The effects of the changes in rail support conditions on the magnitude of the rail bending moments were also investigated [2]. With the continuous development of high-speed railways, polygonal wheel wear and rail corrugation gradually become the nonnegligible issues. The wheel-rail interaction at higher frequencies posed a severe impact on the vehicle and track system because of the wheel-rail wear. When the high-speed trains come along with dynamic loads, the dynamic characteristics of the rail clip are changed, resulting in failure of the $\omega$-type clip, as shown in Figure 2.

Through the past few decades, extensive research studies have been carried out to gain better understandings of the behavior and dynamic features of rail fastenings. In view of the failure of rail clips, dynamic response and material properties are the primary focal areas. For the dynamic 


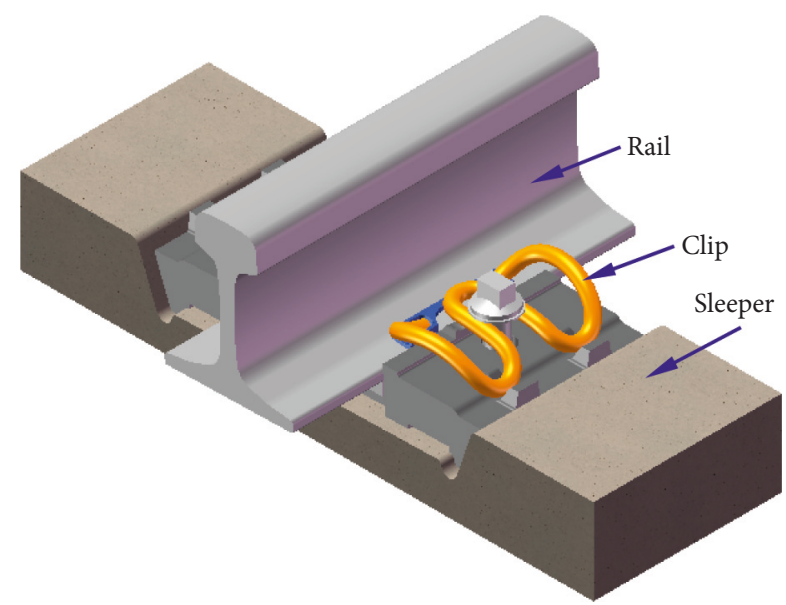

Figure 1: A model of the $\omega$-type fastening.

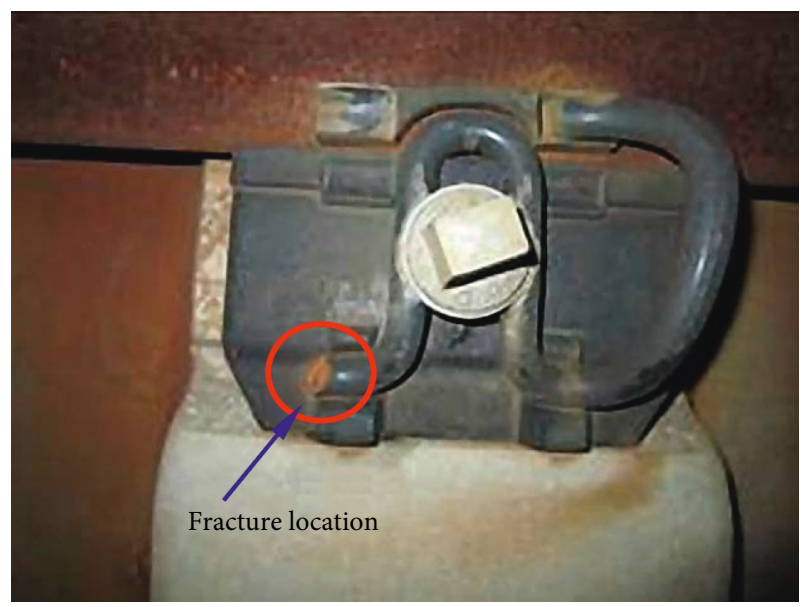

FIGURE 2: $\omega$-type fastening clip failure.

response of the clips, numerical and mechanical models can be built for analyzing dynamic features and the corresponding fatigue limit. Thompson and co-workers measured the vertical and lateral dynamic stiffness of rail fastening systems $[3,4]$. The influence of the excitation magnitude on the stiffness of a specific fastening system was explained. Moreover, irregularities of the rail and wheel, e.g., weldment, wear, and rail corrugation, can contribute to up to $60 \%$ of the wheel load $[5,6]$. Zhao et al. examined the influence of the fastening model on the high-frequency dynamic contact forces at singular rail surface defects [7]. Sadeghi et al. also empirically evaluated the influence of train speeds and axle loads on fatigue of Vossloh and Pandrol flexible clips [8]. It was concluded that the increase of axle loads would cause substantial increases in the plastic deformation of rail clips. Hasap et al. performed a fatigue experiment and finite element analysis of the failure mechanism of an e-clip [9]. They reported that, under normal wheel load, e-clips with high, normal, and low toe loads were run out at $5 \times 10^{6}$ loading cycles, while under the contribution of impact on wheel load, the fatigue life of e-clips was reduced to 5468 and 16839 loading cycles. In addition, the fatigue crack was found to nucleate at the location of highest stress. Zhu et al. found that the vibration response of rail clips on tracks with rail corrugation was about 10 times larger than that of rail clips on tracks without rail corrugation, resulting in the acceleration of clip fatigue damage [10]. Shang et al. numerically evaluated the effect of rail clip vertical displacement on clamping force and stress [11]. It was then suggested that the fatigue crack might easily initiate and propagate in the clip's stress concentration area after repeated passages of trains. Yu et al. discussed the static and fatigue performance of the II fastening clip under different clamping forces and illustrated the stress-strain distribution pattern of the clip [12]. The fatigue life of the fastening clip and the position of the critical fatigue failure point under fatigue loading were also assessed. Xiao et al. systematically investigated fractures of a fastening with the aim of revealing the fracture mechanism and proposing an effective remedy [13]. Mohammadzadeh et al. developed a method for reliability analysis of type SKL14 spring clips [14]. The influence of different variables on the overall probability of failure was studied through sensitivity analysis. Sadeghi et al. studied various track operational conditions against clip permanent deformations [8]. The correlations between clip plastic deformations and track axle loads as well as the train speeds were established. Sadeghi also made a comprehensive field investigation with the aim of achieving a better understanding of the dynamics of prestressed concrete track sleepers [15]. Based on the exploration above, the investigations on vibration behaviors of the railway track systems were attempted [16]. As for the material properties, metallographic tests are commonly used to study the fracture cross section of a failed clip, as well as the chemical components of the clip. In light of the chemical composition of the material, SEM metallographic analysis and hardness testing have been carried out by various researchers, some of whom considered that the fracture of a fastening clip is mainly due to the microstructure deficiencies [17].

Notwithstanding the aforementioned findings, most of the research studies to date are subjected to limited interaction conditions, for instance, the static loading and medium to low frequency range. To accurately and realistically model the loading conditions, properly taking various influential factors into consideration is crucial in generating meaningful outputs, on which the failure mechanism of the rail clips can be investigated. In this work, the $\omega$-type clips in a certain section of the CRH high-speed rail are used to study the failure mechanism with the effect of the wheel-rail interaction. Firstly, a computational model of the vehicle-track coupling system with contact excitation frequencies in the medium to high range is developed. The simulation and experimental tests of the clip modal frequencies with different installation torques are conducted. Secondly, on-site monitoring data are analyzed to not only validate computational model outputs but also examine the high-frequency impacts of wheel-rail wear on clip failure. Moreover, a high-frequency fatigue test machine for rail clips is originally built to further verify the hypothesis of the rail clip failure mechanism. Finally, the design of the 
considered $\omega$-type rail clip is improved according to findings from this study, which avoids the resonant failure of the rail clip.

\section{Computational Model of the Vehicle-Track Interaction and Fastening System}

A computational model of the vehicle-track system, with the consideration of the rail fastening system, was developed to investigate dynamic characteristics of the wheel-rail system and the rail clip.

\subsection{Computational Model of the Vehicle-Track Interaction}

2.1.1. Dynamic Model of the Vehicle-Track Coupling System. The overall stiffness of the fastening system can be derived according to equation (1). As illustrated in the simplified representation in Figure 3, the system stiffness comprises three parts: the stiffness of the fastening $\left(K_{\mathrm{cv}}\right)$, the stiffness of the gauge block $\left(K_{\mathrm{pv}}\right)$, and the stiffness of the base plate $\left(K_{\mathrm{bv}}\right)$. The fastening is in parallel with the gauge under the track in the stiffness calculation, while the bottom buffer pad is arranged in series with them:

$$
K=\frac{K_{\mathrm{bv}}\left(2 K_{\mathrm{cv}}+K_{\mathrm{pv}}\right)}{K_{\mathrm{bv}}+2 K_{\mathrm{cv}}+K_{\mathrm{pv}}} .
$$

2.1.2. Dynamic Response of the Rail. For the past few decades, the train-track coupling dynamics has become the typical approach to study the interaction of the vehicletrack system. It can be used in vehicle structure vibration characteristic analysis and track component fatigue damage evaluation. The operational safety and passenger comfort can also be examined. For instance, the coupling model is able to depict the dynamic performance when the vehicle is passing through a switch or a transition section. In order to study the medium-high frequency vibration component of the fastening system in track sections with rail corrugation, a dynamic model of the vehicle-track coupling system was established, as shown in Figure 4 [18]. The vehicle system is regarded as a multi-rigid-body consisting of the vehicle body, bogie, and wheelset. Five degrees of freedom, namely, vertical, horizontal, nodular, side rolling, and shaking, of the vehicle system determine the vehicle's moving state with the consideration of the nonrailway factors in the vehicle suspension system. The normal force between the wheel and the rail track was calculated by Hertz's nonrailway elastic contact theory, while the tangential creep force was analyzed through Kalker's rail moistening theory, which is followed by a nonrailway correction based on Hertz's theory. The vertical freedom, transversal freedom, and torsional freedom of the left and the right rail track were all properly accommodated. The rail track is regarded as a BernoulliEuler beam supported by elastic points. The distance between supporting points of the rail is the interval space of fastenings. The track plate and supporting layer in the

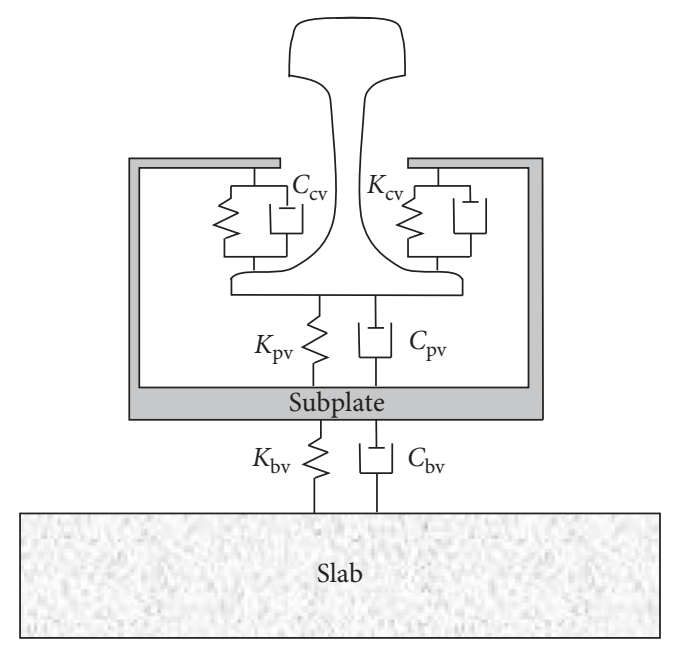

FIgURE 3: Spring dashpot representation of the fastening system.

track structure were the elastic plate of small thickness. The CA mortar layer is regarded as the spring and damping of continuous surface support.

Figure 5 plots the rail vertical response in the time domain. Figure 6 shows the frequency contents of rail vertical response. The response frequencies corresponding to maximum accelerations are found to be $522 \mathrm{~Hz}$ and $603 \mathrm{~Hz}$.

\subsection{FEA of the Fastening System}

2.2.1. FEA Model of the Fastening System. The $\omega$-type railway fastening studied in this paper is a standardized part in the high-speed railway system. As shown in Figure 7, the bolt in the fastening is used to press the middle ring of the clip through the gauge block. The front toes of the clip are used to fix the track root through the insulation block. The heel of the clip is fixed on the track baffle supporter. B is the connection point of the clip and the gauge block. The bolt preload is transmitted to the clip through the gauge block. Meanwhile, the clip and the gauge block are mutually extruded and deformed. As a result, a displacement gap is generated between the gauge block and the clip centre line. The clip is compressed vertically by the bolt preload, which leads to the deformation of the insulation block and the track baffle supporter, as depicted in points A and C. Subsequently, the clip centre line offsets from the insulation block and track baffle supporter. It can be concluded that the effect of the clip offset on the clip vibration of medium-high scale is significant considering the clip resonant modal and the rail corrugation interaction. Therefore, it is needed to analyze the clip vibration modal of high frequencies with respect to the aforementioned relative displacement. Besides, there is a contact stiffness issue between the clip and its matching parts.

The preloading process is simulated by applying pressure on the contact plane between the bolt and the clip. The normal contact is defined using the HARD mode in Abaqus analysis. The Coulomb friction model is used for tangential 


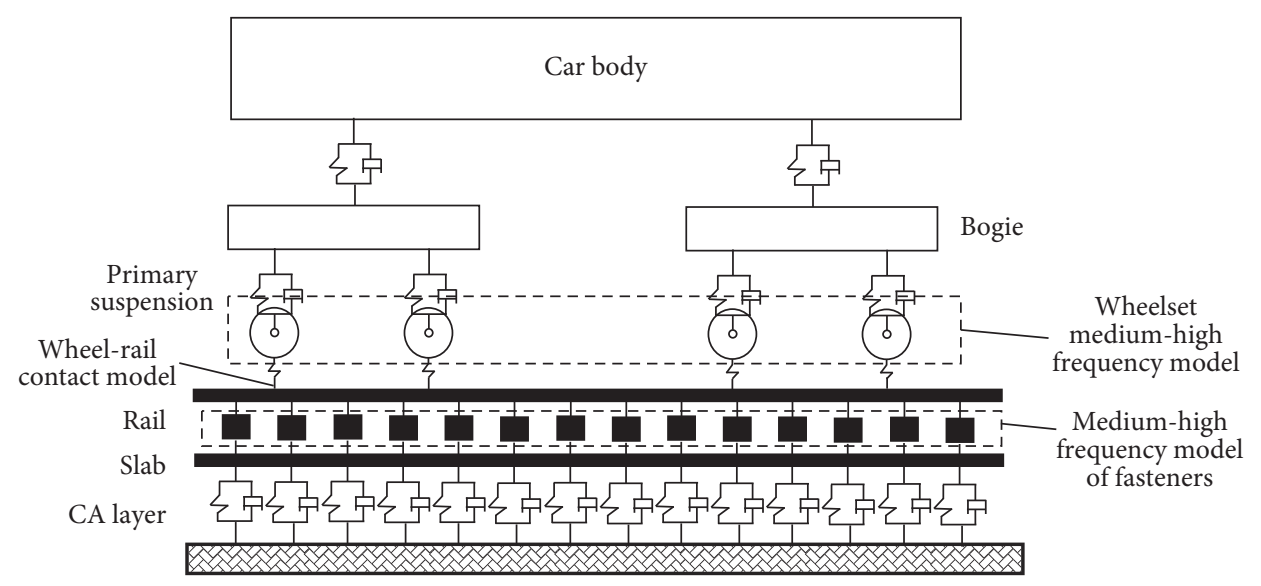

FIGURE 4: Dynamic model of the vehicle-track coupling system.

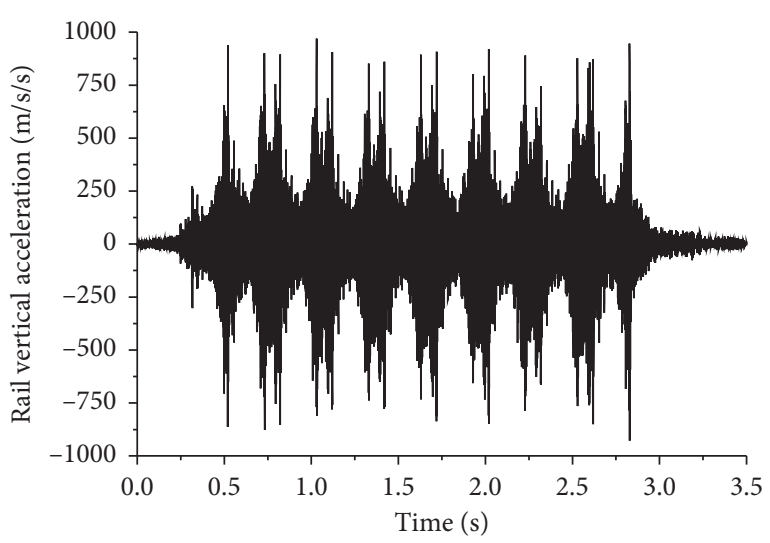

Figure 5: Rail response in the time domain.

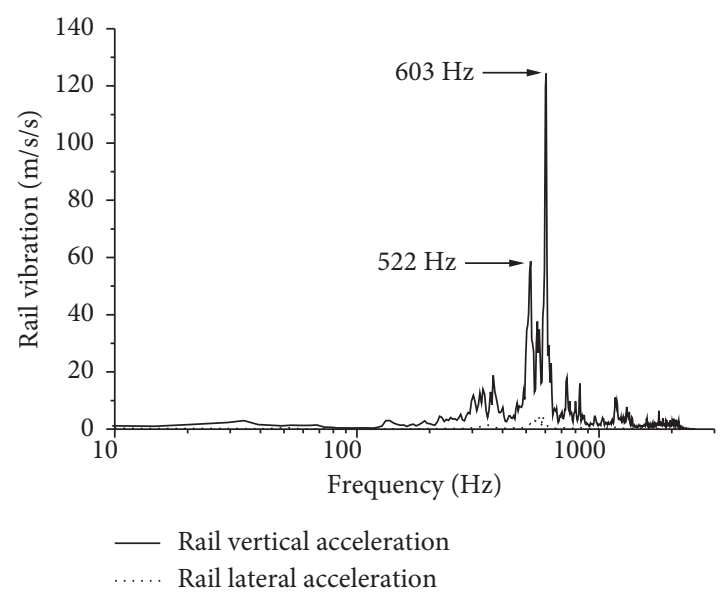

Figure 6: Frequency contents of rail response.

contact, in which the elastic slip is considered between the states of bond and slip. The clip, gauge block, and insulation gasket are all defined with the noncoordinated mode unit C3D8I. The material properties and mesh size used for the FE model are presented in Table 1. The rubber pad under the rail is simulated as the elastic element. The underrail stiffness is $26 \mathrm{kN} / \mathrm{mm}$. The friction coefficient between the

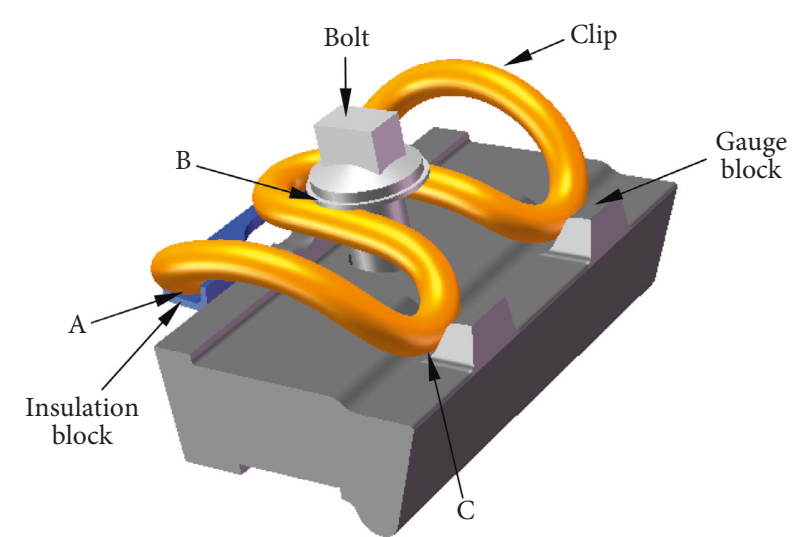

FIgURE 7: Fastening with the $\omega$-type rail clip.

contact surfaces is 0.3 , and the force applied to the middle of the clip is $10 \mathrm{kN}$ to simulate the designed toe load of $10 \mathrm{kN}$.

2.2.2. FE Modal Analysis of the w-Type Rail Clip in Its Installation State. In order to verify the effect of mounting torque of on-site fastenings on the clip vibration, related studies were carried out. The preload of torque $T$ is reversely induced according to the following equation:

$$
T=k_{0} F d,
$$

where $k_{0}$ is the coefficient of preload torque, which is give as 0.2 in this case, $F$ is the preload of the bolt, and $d$ is the bolt nominal diameter. Then, $T$ can be adjusted by changing the value of $F$. The parameter set is shown in Table 2. An FEA model of the fastening was constructed, as shown in Figure 8. Mounting torque with varying values was applied to the rail clip to realistically model its on-site conditions. Modal frequencies of the first five vibration modes of the rail clip with the bolt mounting torque varying from $100 \mathrm{~N} \cdot \mathrm{m}$ to $300 \mathrm{~N} \cdot \mathrm{m}$ are plotted in Figure 9. For the $1^{\text {st }}$ and $3^{\text {rd }}$ modes, modal frequencies increase with increasing torque values, whereas for the rest of the vibration modes, modal frequencies remain more or less the same with varying torque values. This 
TABLE 1: Material parameter set.

\begin{tabular}{lccccc}
\hline Components & Elastic modulus $(\mathrm{MPa})$ & Poisson's ratio & Element type & Mesh size $(\mathrm{mm})$ & $\begin{array}{l}\text { Density } \\
\left(\mathrm{kg} / \mathrm{m}^{3}\right)\end{array}$ \\
\hline Rail & $2.10 e 5$ & 0.3 & C3D8I & 10 & 7800 \\
Clip & $2.10 e 5$ & 0.3 & C3D8I & 0.5 & 7800 \\
Insulation block & $2.4 e 3$ & 0.4 & C3D8I & 0.5 & 1000 \\
Gauge block & $2.4 e 3$ & 0.4 & C3D8I & 3 \\
\hline
\end{tabular}

TABle 2: Parameter set.

\begin{tabular}{lcccc}
\hline Mechanical properties & \multicolumn{4}{c}{ Parameter } \\
\hline Torque $(\mathrm{N} \cdot \mathrm{m})$ & 100 & 150 & 200 & 250 \\
Preload of the bolt $(\mathrm{kN})$ & 20 & 22.9 & 27 & 300 \\
Clamp force of the clip $(\mathrm{kN})$ & 8.2 & 9.1 & 11.5 & 33 \\
\hline
\end{tabular}

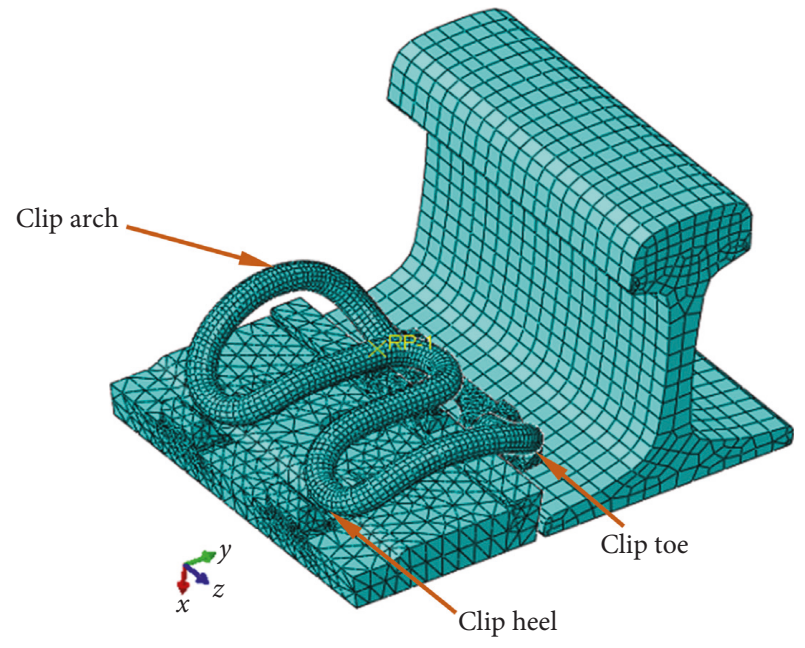

Figure 8: FEA model of fastening with the $\omega$-type clip.

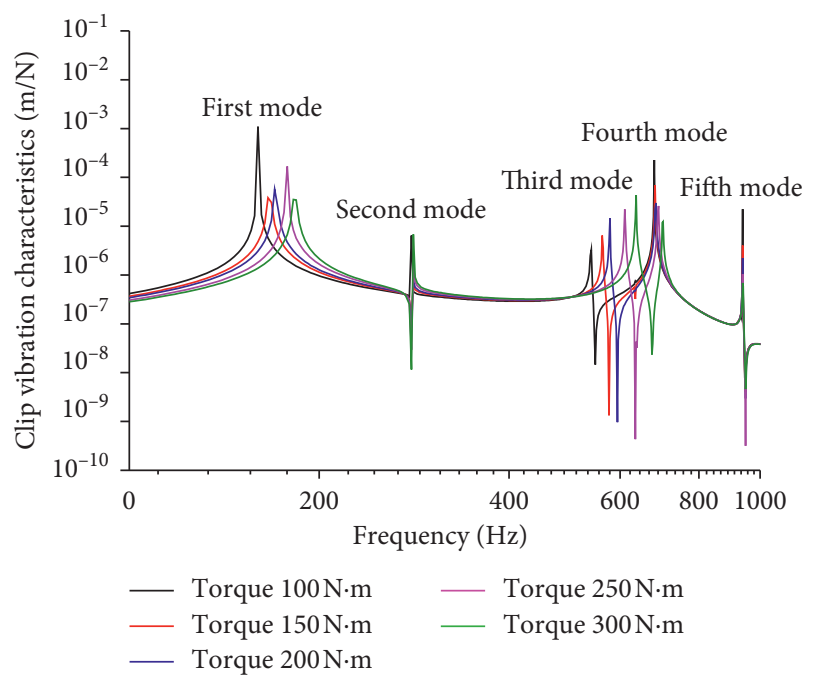

FIGURE 9: Simulated results of the clip modal characteristics.

observation is associated with the boundary conditions and their high energy concentration in the clip heel area as shown in the mode shapes of the rail clips, as shown in Table 3.

Furthermore, it is found that the maximum response frequency of the rail track coincides precisely with the $3^{\text {rd }}$ modal frequency of the rail clip with $250 \mathrm{~N} \cdot \mathrm{m}$ bolt torque. It can therefore be obtained that the clip failure could be due to the resonance of the wheel-rail contact wear and the $3^{\text {rd }}$ order modal response of the rail clip. At the third modal frequency, the clip arch is symmetrically deformed upside down around the toe and the heel because of the rail vertical vibration, which looks like a butterfly waving wings. This coincides with the conclusion in reference [10] which believes that the stress concentration occurs at the clip heel support point. The periodic motion or highfrequency excitation would lead to fatigue fracture or nonnormal damage at the clip heel. The proposed work mainly focused on the third order of the butterfly waving wing modal in the exploration of different installation conditions.

2.2.3. Experimental Modal Analysis of the w-Type Rail Clip in Its Installation State. In order to verify the accuracy of the simulation results in Section 2.2.2, the assembly test was carried out to study the $\omega$-type rail clip. Two accelerometers were placed on the left and the right arm of the clip, respectively. The hammer test was carried out for modal identification, as shown in Figure 10. The assembly modal response of the clip is tested by applying different torques to the bolt. The results are shown in Table 4. Through the comparison and analysis of the test, it was found that the average error between the simulation output and the test results was $2.8 \%$, which confirmed the clip simulation model.

\section{Dynamic Parameter Identification of the Clip}

A real-time vibration monitoring of the same system was undertaken on a high-speed nonballasted railway site. The rail clips on-site were the same as the ones built in the computational model in Section 2. Real-time data were collected and analyzed not only for validation purpose but also for assessment of the impacts of wheel polygonalisation and rail corrugation on rail clip failure, which will be discussed in the following sections. The test section is located in the China Wuhan-Guangzhou high-speed railway with a curve radius of $10000 \mathrm{~m}$, a superelevation of $125 \mathrm{~mm}$, and a 
TABLE 3: Modal frequency and mode shape of the rail clip.

\begin{tabular}{lcll}
\hline Mode & Bolt force $(\mathrm{kN})$ & Clip frequency $(\mathrm{Hz})$ & Mode shape \\
First order & & \\
$20 \sim 33$ & $160 \sim 182$
\end{tabular}

Second order

20 33

286

$U$, magnitude

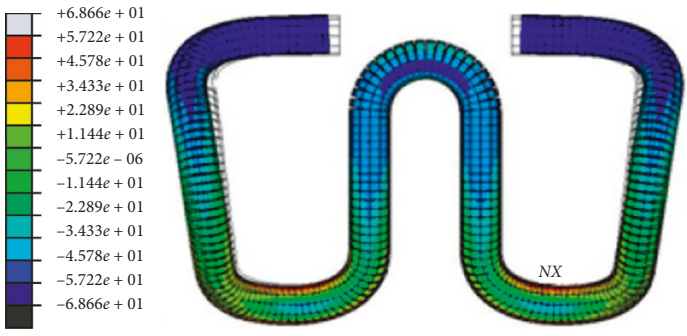

Third order

20 33

520 636
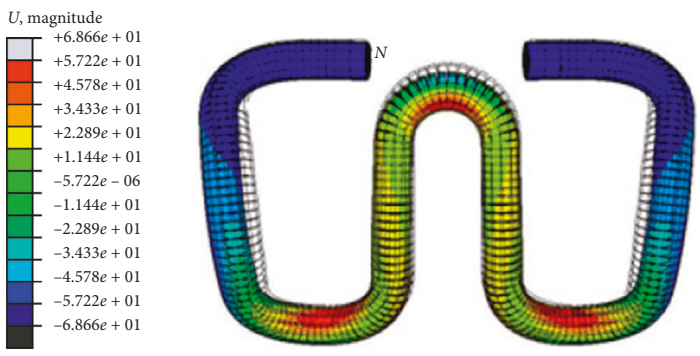

Fourth order

20 33

680 702
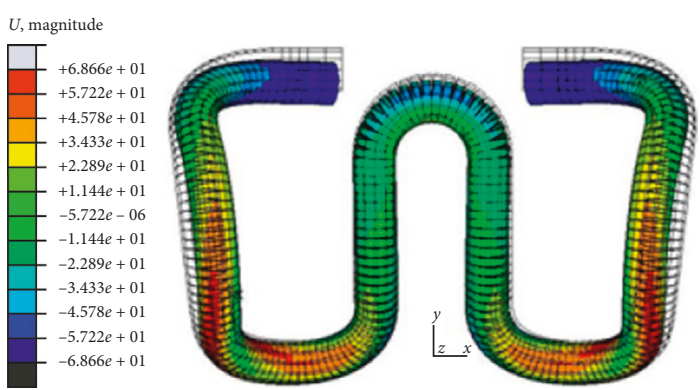

Fifth order

20 33

934

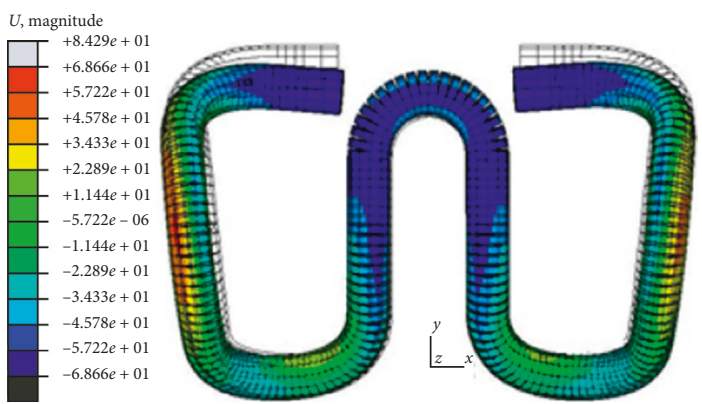




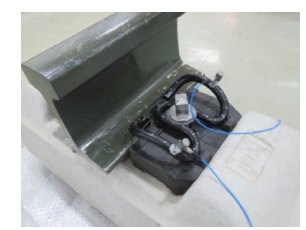

FIgURe 10: Assembly modal test of the clip.

slope of $5.2 \%$. The ballastless track bed is a CRTS-II platetype double-block structure. The test vehicle is of CRH3 type with a speed of $300 \mathrm{~km} / \mathrm{h}$.

3.1. Wheel Polygonalisation. Wheel polygonalisation intensifies the dynamic impact of the wheel-rail interaction. In the cases of high operational speed, such an impact could potentially damage the vehicle and track components. Based on measured data and a statistical summary of a standard high-speed train $\mathrm{CRH} 3$, it can be obtained that the wheel more or less resembles an octadecagon (an 18-edge polygon) (Figure 11), and wheel wear is one of the dominant issues of the existing railway system $[19,20]$.

When the train is in operation, the wheels are subjected to periodic excitation which comes from inherent characteristics of the vehicle-track system. The uneven wear frequency could be presented as

$$
f=\frac{v}{\lambda}=\frac{v}{\pi D} \times n
$$

where $f$ is the excitation frequency of the wheel polygonalisation, $v$ is the train speed, $D$ is the wheel diameter, and $n$ is the number of wheel wear orders. According to the above equation, the excitation frequency of the CRH3 wheel is about $569 \mathrm{~Hz}$, which again falls within the $3^{\text {rd }}$ order modal frequency range of the $\omega$-type clip.

3.2. Rail Corrugation. The rail corrugation takes place in both curved and straight railway sections. The wavelength is distributed in the range of $151 \mathrm{~mm}$, as shown in Figure 11. When the train running speed is $250-350 \mathrm{~km} / \mathrm{h}$, the excitation frequency of rail corrugation is in the range of $463 \mathrm{~Hz}-1389 \mathrm{~Hz}$. Thus, the response of corrugation induced by wheel-rail contact force is classified as medium- to highfrequency excitation [21-24]. The wheel-rail interaction subsequently leads to medium- to high-frequency vibration within the fastening system. From on-site measurements, when the train running speed is $300 \mathrm{~km} / \mathrm{h}$, the frequencies of maximum track excitation are $370 \mathrm{~Hz}$ and $550 \mathrm{~Hz}$, which correspond to wavelengths of $225.3 \mathrm{~mm}$ and $151.4 \mathrm{~mm}$, as shown in Figure 12.

3.3. Wheel-Rail Contact Force. The rail corrugation intensifies the wheel-rail interaction. It could potentially cause damage of vehicle and track components in severe cases. When the train running speed is $300 \mathrm{~km} / \mathrm{h}$, the wheel-rail vertical contact force is measured and plotted in Figure 13. The excitation frequencies of maximum vertical force are found to be $522 \mathrm{~Hz}$ and $605 \mathrm{~Hz}$, and the latter coincides with the $3^{\text {rd }}$ modal frequency of the rail clip.

3.4. Dynamic Analysis and Damage Mechanism of the Fastening Clip. As demonstrated in the previous two sections, the excitation frequency of both wheel polygonalisation and rail corrugation falls within the range of the $3^{\text {rd }}$ modal frequency of the rail clip. Furthermore, the frequencies of maximum wheel-rail force agree well with the dynamic frequencies of rail clips, as shown in Figure 14. In other words, the failure mechanism of the $\omega$-type rail clip in this study is due to wheel-rail wear induced by the resonant vibration at the $3^{\text {rd }}$ modal frequency of the clip.

\section{Fatigue Damage Test of the Clip}

4.1. Test Setup. A dynamic fatigue test of the fastening clip under high-frequency excitation is performed to verify the failure mechanism of the $\omega$-type clip. The excitation interaction at high frequencies for modeling the on-site wheelrail interaction force needs to be incorporated into the test. As shown in Figure 15, a novel fatigue test system was designed, which allows a maximum loading frequency of $1200 \mathrm{~Hz}$. In addition, the test frame is able to accommodate different types of rail clips, e.g., $\omega$-type and e-type clips.

For the amplitude characteristics of the loading clip, this design adopts the camshaft structure of different diameters and different polygon order numbers. The diameter of the camshaft is $\varphi$, the polygon order is $n$, the distance between the clip burke points to the fixed end of the linkage is $l_{1}$, and the distance between the clip burke points to the linkage free end is $l_{2}$, as shown in Figure 13. Therefore, the camshaft amplitude $a_{1}$ and the clip amplitude $a_{2}$ can be expressed as follows:

$$
\begin{aligned}
& a_{1}=\frac{\varphi}{2}\left(1-\cos \frac{180}{n}\right), \\
& a_{2}=\frac{l_{1}}{l_{1}+l_{2}} \cdot a_{1}=\frac{l_{1}}{l_{1}+l_{2}} \cdot \frac{\varphi}{2}\left(1-\cos \frac{180}{n}\right) .
\end{aligned}
$$

4.2. Dynamic Fatigue Test of the $\omega$-Type Clip. The $\omega$-type clip was fixed onto the clamping block with a bolt torque of $250 \mathrm{~N} \cdot \mathrm{m}$. The clip installation modal frequency was $590 \mathrm{~Hz}$, which is fixed and tested by the fatigue system, as shown in Figure 16, based on which the motor speed was set to $1420 \mathrm{rpm}$ by adjusting the transmission ratio and the wavelength number of the camshaft. During the dynamic high-frequency fatigue test, the clip vertical displacement was merely $0.05 \mathrm{~mm}$ measured by a laser displacement sensor. However, the corresponding vibration amplitude was $3.3 \mathrm{~g}$.

The $\omega$-type clip in the test fractured after 125 minutes of continuous operation, as shown in Figure 17. The total cycles of this test are equivalent to about 4.425 million. The result 


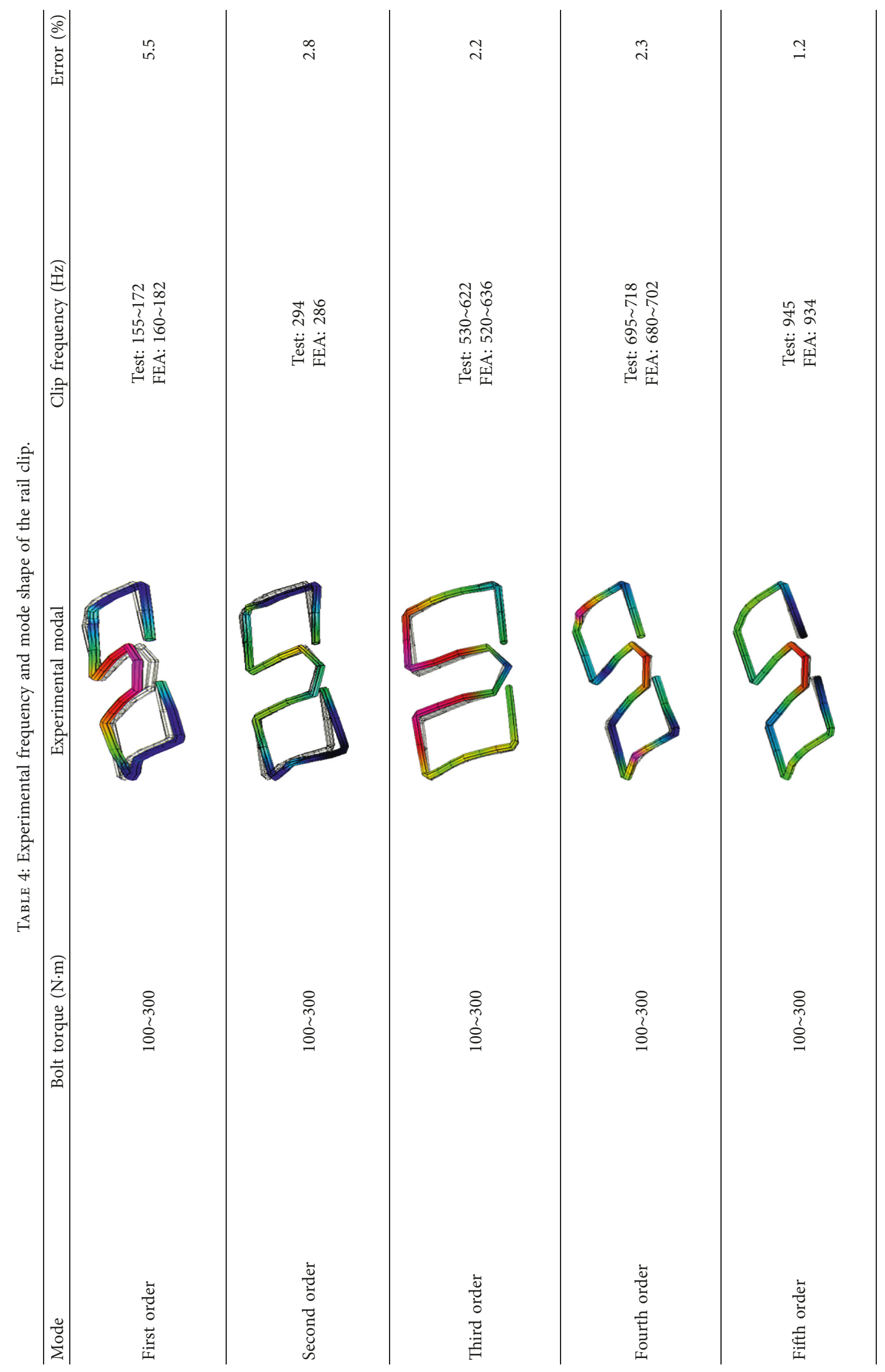




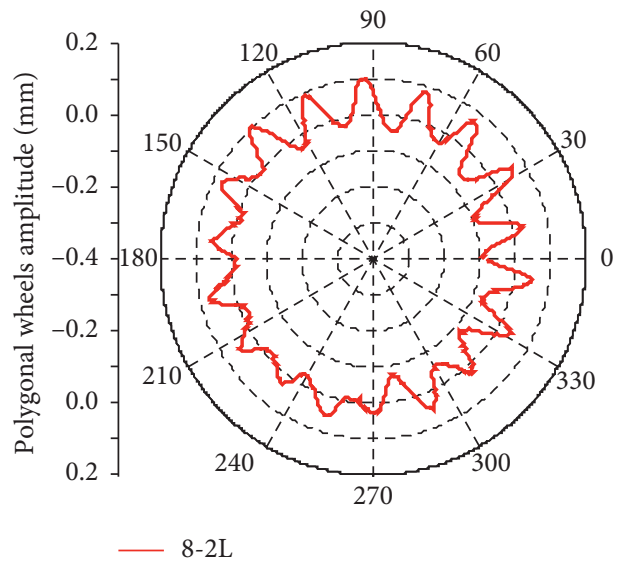

FIGURE 11: CRH3 wheel polygonalisation.

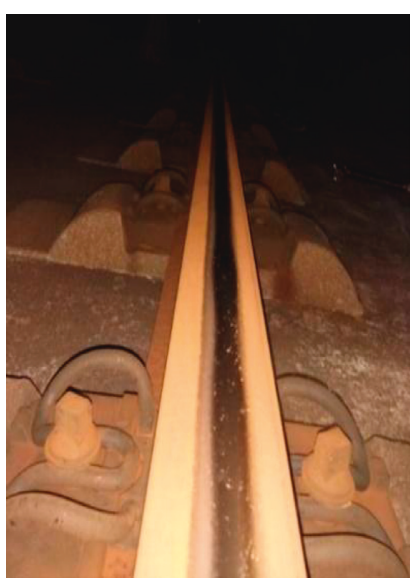

(a)

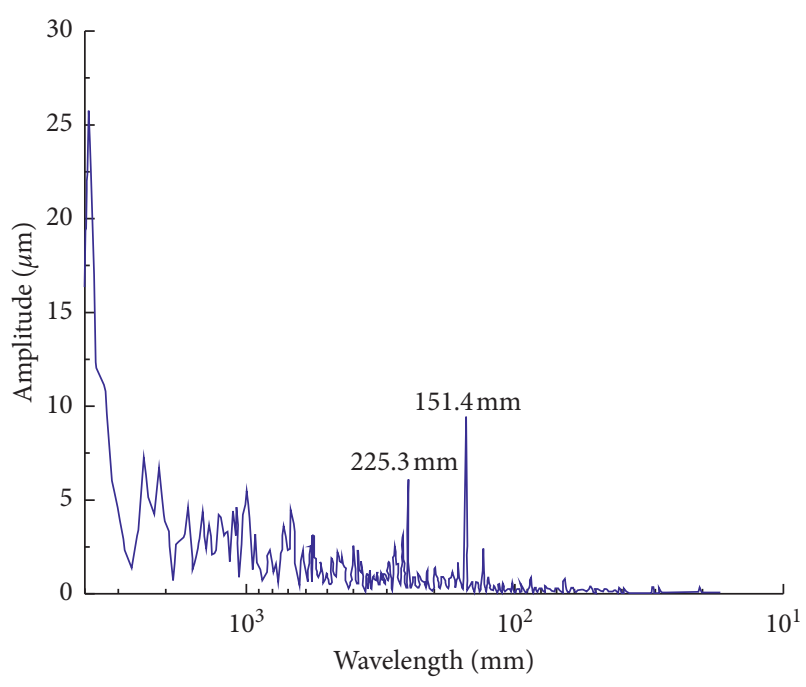

(b)

FIGURE 12: On-site test (a) and output (b) of the rail corrugation in the high-speed railway.

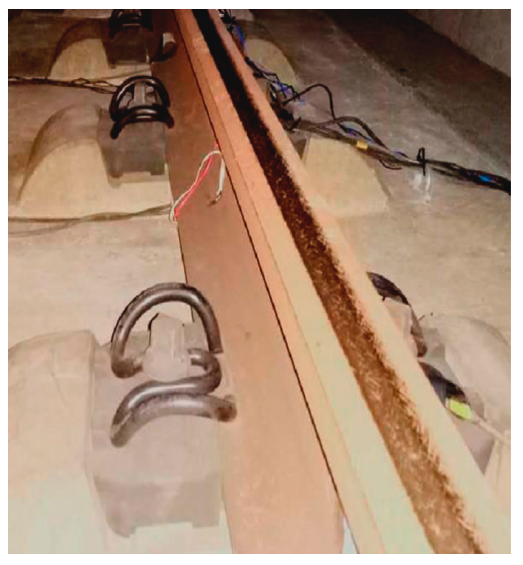

(a)

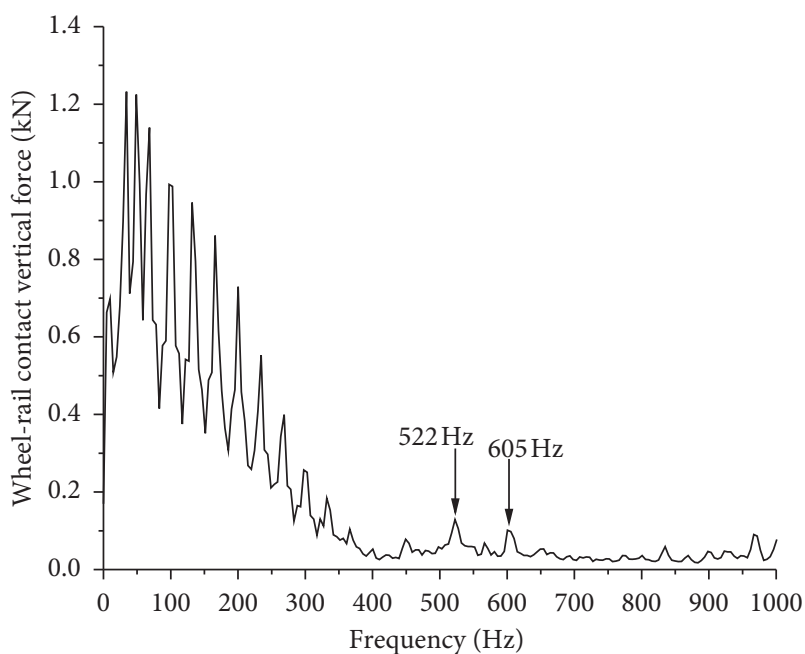

(b)

FIGURE 13: On-site test (a) and output (b) of wheel-rail vertical contact force. 


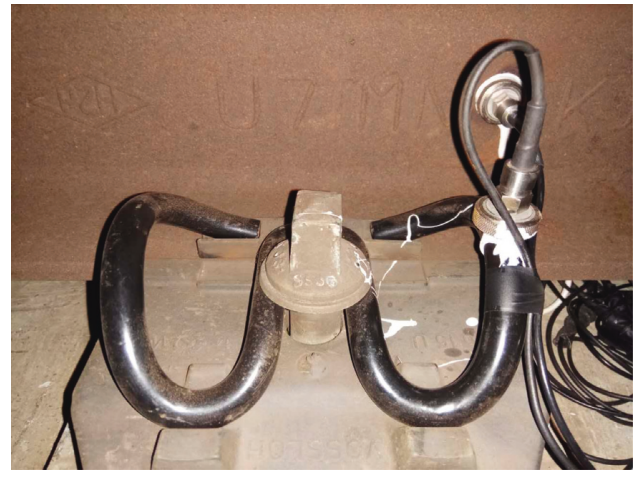

(a)

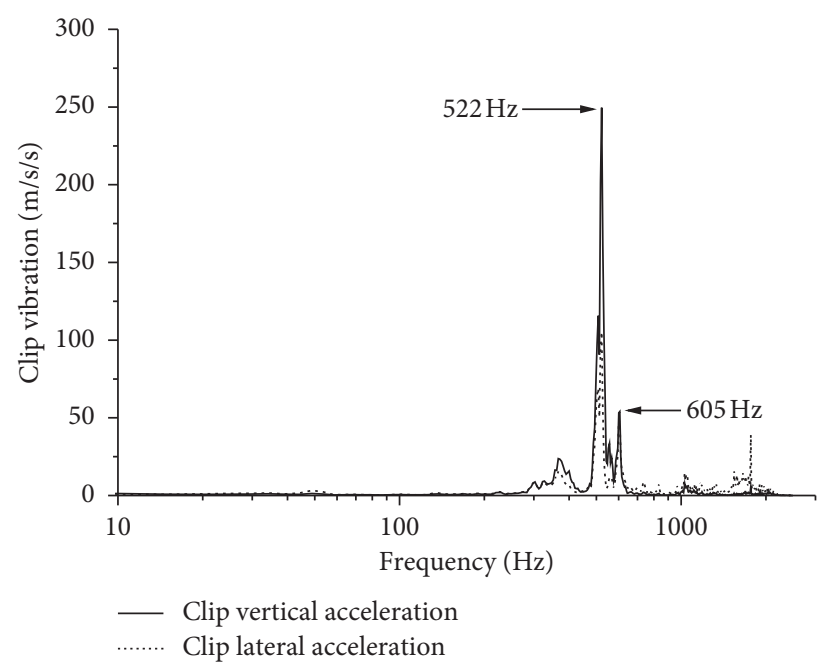

(b)

FIGURE 14: On-site test (a) and output (b) of frequency contents of rail clip vibration.
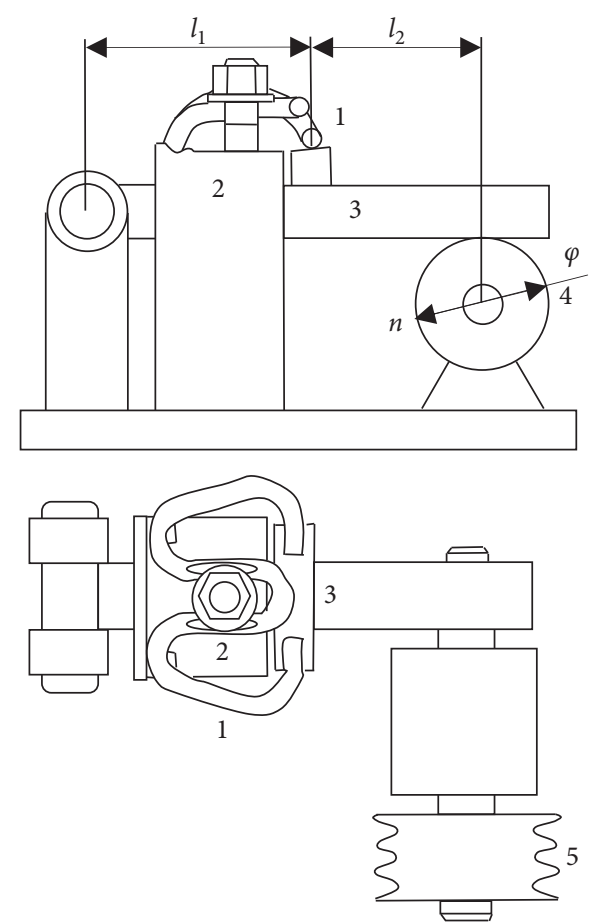

(1) Rail clip

(2) Clip clamping block

(3) Loading linkage

(4) Loading camshaft

(5) Frequency-adjustable motor

FiguRE 15: High-frequency fatigue test system for rail clips.

further verifies that the failure mechanism of the rail clips is the coincidence of the wheel-rail wear excitation and the $3^{\text {rd }}$ modal frequency of the clip.

\section{Structural Improvement of the $\omega$-Type Clip}

5.1. Structural Improvement of the Clip Design. The study so far demonstrated that $\omega$-type rail clip failure was due to the resonant vibration of wheel-rail wear excitation and modal frequency of the clip's $3^{\text {rd }}$ mode. Altering stiffness and mass of the clip could shift the modal frequency of the $3^{\text {rd }}$ mode, thus avoiding resonance. The equivalent stiffness and the equivalent mass are key factors to determine the clip resonant modes in terms of stress conditions and modal characteristics. In this particular case, the equivalent stiffness needs to be increased and equivalent mass needs to be 


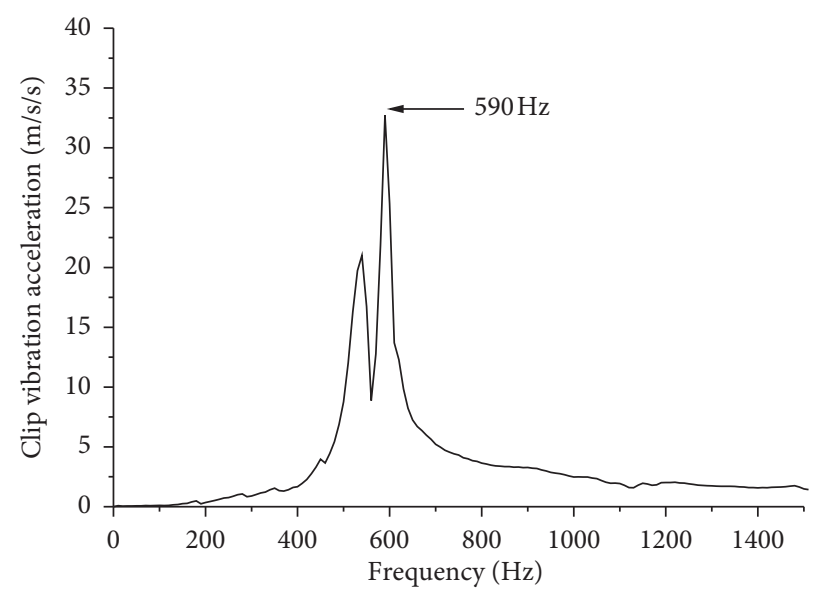

FIGURE 16: Installation modal frequency of the $\omega$-type clip.

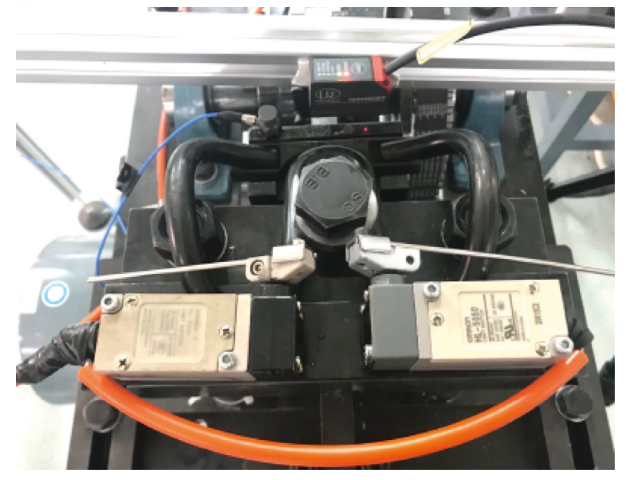

(a)

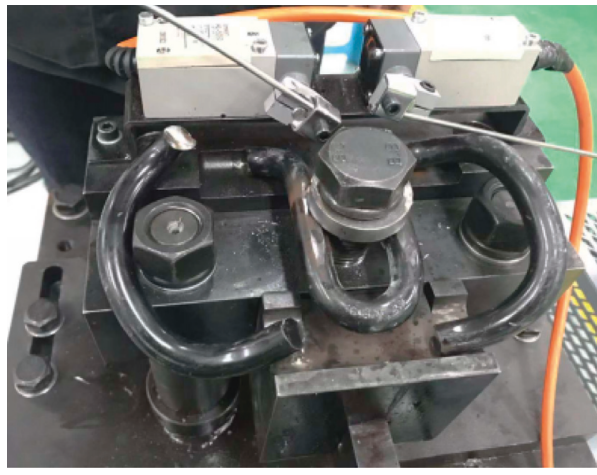

(b)

FIgURE 17: $\omega$-type clip broken after 125 minutes.

reduced. To match the original installation requirement, the equivalent mass can be adjusted by altering the diameter of the rail clip. A reduction in the overall vibration mass of the clip can be achieved by changing the configuration, e.g., curvature, of the back wing and the arch of the clip. Meanwhile, the clip diameter was increased from $15 \mathrm{~mm}$ to $15.5 \mathrm{~mm}$. And the overall mass of the clip was decreased from $0.85 \mathrm{~kg}$ to $0.79 \mathrm{~kg}$, as shown in Table 5 . Figure 18 shows a direct comparison of the clip design before and after the improvement.

5.2. Modal Characteristics of the Improved $\omega$-Type Rail Clip. According to the improved design, a $\omega$-type rail clip, which is shown in Figure 19, was built. A modal analysis was performed to compare modal characteristics of the rail clip before and after design improvement. The rail clips were installed on two adjacent sides of a rail to test their boundary conditions and the modal characteristics. The installation conditions of the track remain the same. Modal frequencies of the original and improved $\omega$-type rail clips are shown in Table 6. None of the modal frequency of the rail clip is in line with the frequency of the wheel-rail excitation force or with that of wheel polygonalisation or rail corrugation. The original $2^{\text {nd }}$ frequencies shifted from $598 \mathrm{~Hz}$ to $875 \mathrm{~Hz}$. Therefore, resonance is successfully prevented.

5.3. Frequency Response of the Improved w-Type Rail Clip. An experiment, as shown in Figure 20, was undertaken to obtain the frequency response of the improved rail clip with the effect of wheel-rail coupling. Results of the test are summarized and plotted in Figure 21. It can be found that the modal frequencies of the two clips basically coincide at $480 \mathrm{~Hz}$ with the effect of the wheel-rail coupling. The $2^{\text {nd }}$ and $3^{\text {rd }}$ resonant frequencies of the original clip are $585 \mathrm{~Hz}$ and $625 \mathrm{~Hz}$, respectively, whereas for the improved rail clip, the counterparts are shifted to $886 \mathrm{~Hz}$ and $1050 \mathrm{~Hz}$, respectively, thus successfully moving away from the excitation frequency of the wheel-rail interaction and preventing the clip from resonant vibration.

\section{Conclusions}

In this paper, the failure mechanism of a $\omega$-type fastening clip in the $\mathrm{CRH}$ high-speed railway was studied. A 
TABle 5: Design parameters of the $\omega$-type rail clip before and after improvement.

\begin{tabular}{lcccccc}
\hline Clip size & Length $(\mathrm{mm})$ & Width $(\mathrm{mm})$ & Height $(\mathrm{mm})$ & Diameter $(\mathrm{mm})$ & Mass $(\mathrm{kg})$ & Stiffness $(\mathrm{kN} / \mathrm{mm})$ \\
\hline Original & 176 & 119 & 51 & 15 & 0.85 & 0.67 \\
Improved & 168 & 121 & 38 & 15.5 & 0.79 & 0.98 \\
\hline
\end{tabular}

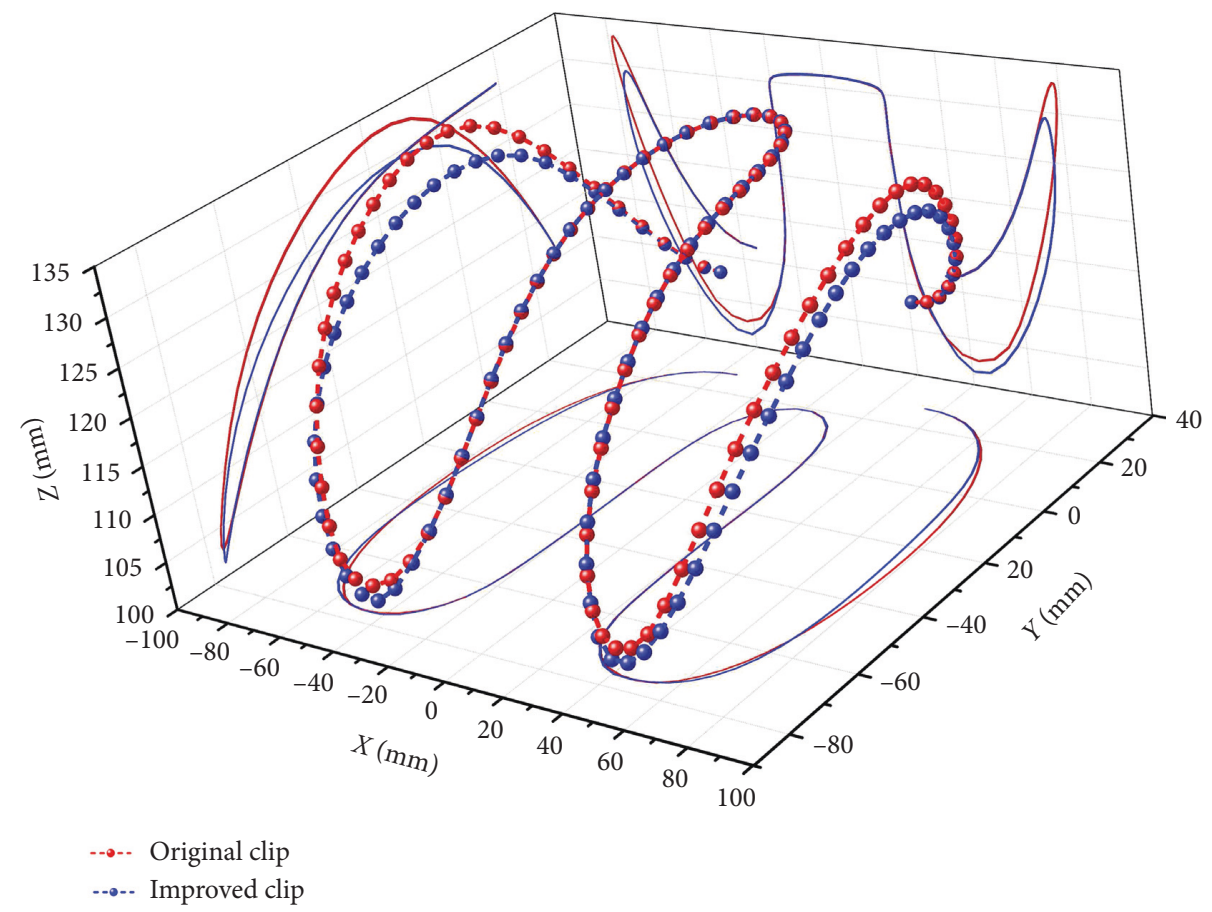

FIgURE 18: Configuration of the $\omega$-type clip before and after structural improvement.

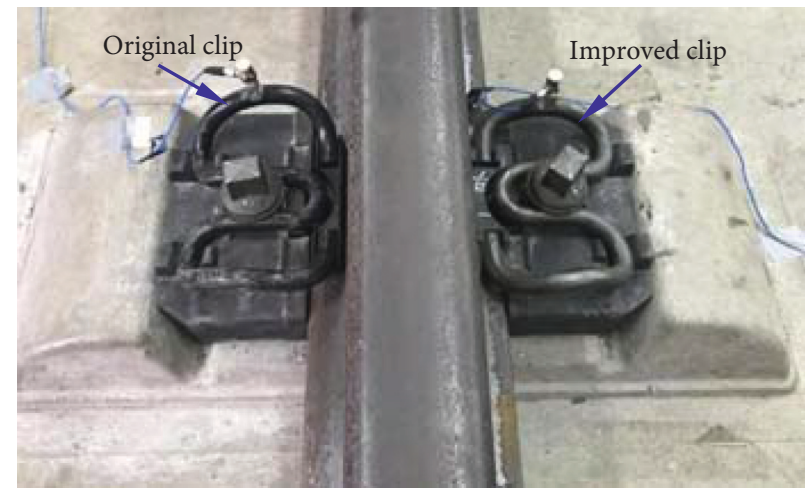

FIGURE 19: Original and improved $\omega$-type rail clips in the test.

TABle 6: Modal frequencies of the original and improved rail clips.

\begin{tabular}{lccc}
\hline Order & Original clip $(\mathrm{Hz})$ & Improved clip $(\mathrm{Hz})$ & Variation rate $(\%)$ \\
\hline 1 & 483 & 496 & 3.1 \\
2 & 598 & 875 & 32.7 \\
3 & 625 & 998 & 37.4 \\
4 & 906 & 1290 & 29.8 \\
5 & 1251 & 1415 & 11.6 \\
\hline
\end{tabular}




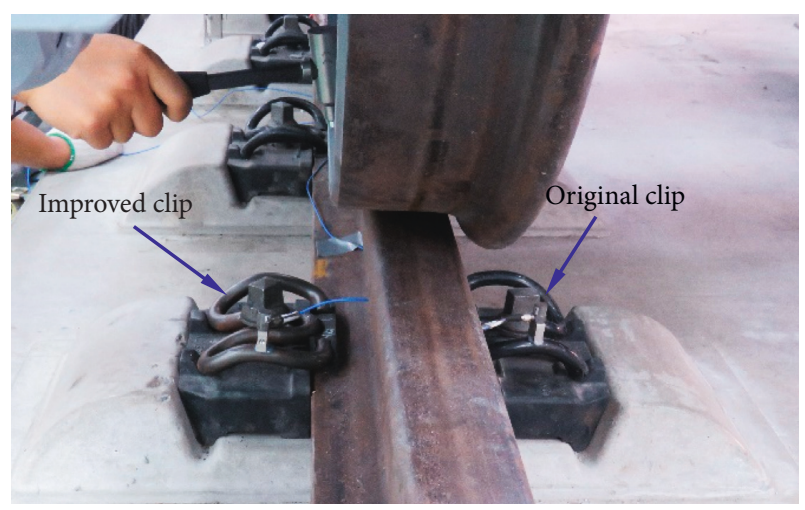

Figure 20: Frequency response test of the fastening clip before and after improvement.

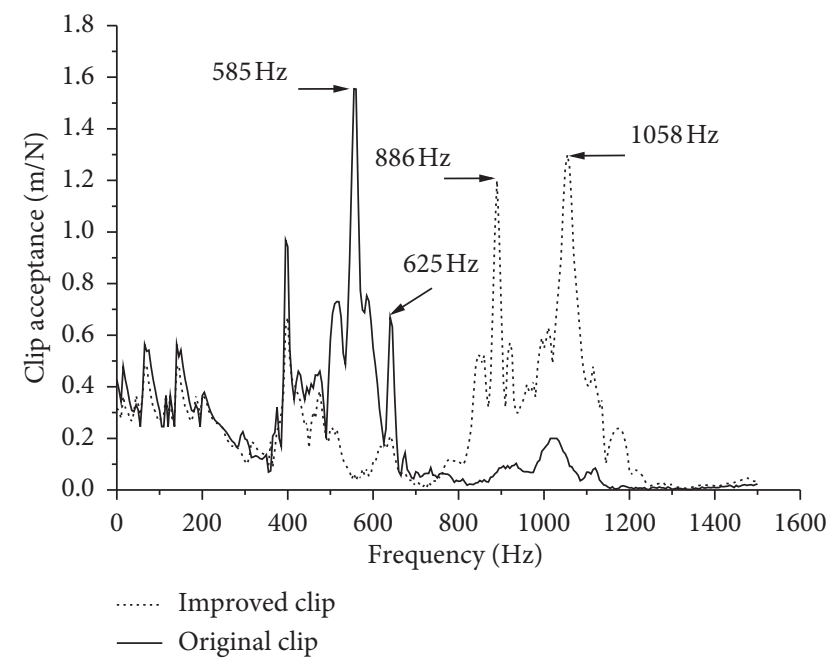

FIgURE 21: Frequency contents of the response test.

computational model of the wheel-rail coupling system, taking fastenings into consideration, was developed. Realtime vibration monitoring of the same clip on a CRH highspeed rail line was carried out for validation purpose. On-site measurement was also taken to study the effects of wheel polygonalisation and rail corrugation. A novel high-frequency fatigue test system for the rail clips was designed and constructed to conduct experimental verification of the clip failure mechanism hypothesis. Preliminary results can be concluded as follows:

(1) Through the dynamic wheel-rail coupling model, the main peaks of the frequency response of the track are $522 \mathrm{~Hz}$ and $603 \mathrm{~Hz}$. Modal frequency of the $3^{\text {rd }}$ vertical mode of the $\omega$-type clip is in the range between $520 \mathrm{~Hz}$ and $636 \mathrm{~Hz}$.

(2) Wheel polygonalisation and rail corrugation intensify the wheel-rail interaction. Excitation frequency of the wheel-rail interaction force coincides with the $3^{\text {rd }}$ modal frequency of the rail clip, causing resonance, which is the failure mechanism of the rail clip in consideration.
(3) A novel high-frequency fatigue test system for rail clips was designed to conduct the corresponding experimental test. The experimental results further verified the failure mechanism suggested via the computational model and on-site monitoring data.

(4) The $\omega$-type rail clip was structurally improved according to findings from this study. Modal frequencies of the rail clip were shifted to well above $800 \mathrm{~Hz}$, which avoided the resonant vibration.

\section{Data Availability}

The data used to support the findings of this study are available from the corresponding author upon request.

\section{Conflicts of Interest}

The authors declare that they have no conflicts of interest.

\section{Acknowledgments}

This research project was supported by joint funds from both China Railway Cooperation and National Natural Science Foundation of China (Grant No. U1834201).

\section{References}

[1] J. Sadeghi and P. Barati, "Evaluation of conventional methods in analysis and design of railway track system," International Journal of Civil Engineering, vol. 8, no. 1, pp. 44-56, 2010.

[2] J. Sadeghi and F. Hashemi, "Influences of rail support conditions on mechanical behavior of railway track system," Transactions of the Canadian Society for Mechanical Engineering, vol. 32, no. 3-4, pp. 561-574, 2008.

[3] J. Ryue, D. J. Thompson, P. R. White, and D. R. Thompson, "Investigations of propagating wave types in railway tracks at high frequencies," Journal of Sound and Vibration, vol. 315, no. 1-2, pp. 157-175, 2008.

[4] D. J. Thompson and J. W. Verheij, "The dynamic behaviour of rail fasteners at high frequencies," Applied Acoustics, vol. 52, no. 1, pp. 1-17, 1997.

[5] L. Ling, W. Li, H. Shang, X. Xiao, Z. Wen, and X. Jin, "Experimental and numerical investigation of the effect of rail corrugation on the behaviour of rail fastenings," Vehicle System Dynamics, vol. 52, no. 9, pp. 1211-1231, 2014.

[6] S. Mohammadzadeh, S. Ahadi, and M. Nouri, "Stress-based fatigue reliability analysis of the rail fastening spring clipunder traffic loads," Latin American Journal of Solids and Structures, vol. 11, no. 6, pp. 993-1011, 2014.

[7] X. Zhao, Z. Li, and R. Dollevoet, "Influence of the fastening modeling on the vehicle-track interaction at singular rail surface defects," Journal of Computational and Nonlinear Dynamics, vol. 9, no. 3, pp. 1-11, 2014.

[8] J. Sadeghi, M. Fesharaki, and A. Khajehdezfuly, "Influences of train speed and axle loads on life cycle of rail fastening clips," Transactions of the Canadian Society for Mechanical Engineering, vol. 39, no. 1, pp. 1-11, 2015.

[9] A. Hasap, P. Paitekul, N. Noraphaiphipaksa, and C. Kanchanomai, "Analysis of the fatigue performance of elastic rail clip," Engineering Failure Analysis, vol. 92, pp. 195-204, 2018. 
[10] S. Y. Zhu, C. B. Cai, and Y. Qiang, "Dynamic analysis of rail fastening clip in high-speed railway," Journal of Engineering Mechanics, vol. 6, pp. 254-258, 2013, in Chinese.

[11] H. X. Shang, Z. F. Wen, L. Wu, W. Li, S. F. Zhang, and X. S. Jin, "Finite element analysis of type iii rail fastening clip failure in metro railway," Engineering Mechanics, vol. 32, pp. 210-215, 2015, in Chinese.

[12] Z. R. Yu, Y. Yuan, Y. Q. Zhang et al., "Fatigue properties of elastic bars of fastening systems installed with high-speed railways," Journal of the China Railway Society, vol. 36, no. 7, pp. 90-95, 2014, in Chinese.

[13] H. Xiao, J.-B. Wang, and Y.-R. Zhang, "The fractures of e-type fastening clips used in the subway: theory and experiment," Engineering Failure Analysis, vol. 81, pp. 57-68, 2017.

[14] S. Mohammadzadeh, S. Ahadi, and H. Keshavarzian, "Assessment of fracture reliability analysis of crack growth in spring clip type vossloh SKL14," Proceedings of the Institution of Mechanical Engineers, Part O: Journal of Risk and Reliability, vol. 228, no. 5, pp. 460-468, 2014.

[15] J. Sadeghi, "Field investigation on vibration behavior of railway track systems," International Journal of Civil Engineering, vol. 8, no. 3, pp. 232-241, 2010.

[16] J. Sadeghi, "Field investigation on dynamics of railway track pre-stressed concrete sleepers," Advances in Structural Engineering, vol. 13, no. 1, pp. 139-151, 2010.

[17] W. X. Wang, Z. Wang, L. Zhao et al., "Failure analysis of SKL15 spring fasteners for high-speed railway," Heat Treatment of Metals, vol. 12, pp. 108-112, 2013, in Chinese.

[18] J. Martínez-Casas, J. Giner-Navarro, L. Baeza, and F. D. Denia, "Improved railway wheelset-track interaction model in the high-frequency domain," Journal of Computational and Applied Mathematics, vol. 309, pp. 642-653, 2017.

[19] X. Liu and W. Zhai, "Analysis of vertical dynamic wheel/rail interaction caused by polygonal wheels on high-speed trains," Wear, vol. 314, no. 1-2, pp. 282-290, 2014.

[20] R. Bogacz and K. Frischmuth, "On dynamic effects of wheelrail interaction in the case of polygonalisation," Mechanical Systems and Signal Processing, vol. 79, pp. 166-173, 2016.

[21] J. H. Xiao, Z. Q. Yan, and Y. H. Tu, "On impact of wheel-rail vibration on HSR fastener damage," Chinese Railways, vol. 11, pp. 10-14, 2017, in Chinese.

[22] Z. Q. Jiang, D. L. Si, and W. Li, "On rail corrugation of highspeed railway," China Railway Science, vol. 35, no. 4, pp. 9-14, 2014, in Chinese.

[23] S. L. Grassie, "Rail corrugation: advances in measurement, understanding and treatment," Wear, vol. 258, no. 7-8, pp. 1224-1234, 2005.

[24] S. L. Grassie, "Rail corrugation: characteristics, causes and treatments," Proceedings of the Institution of Mechanical Engineers, Part F: Journal of Rail and Rapid Transit, vol. 223, no. 6, pp. 581-596, 2009. 


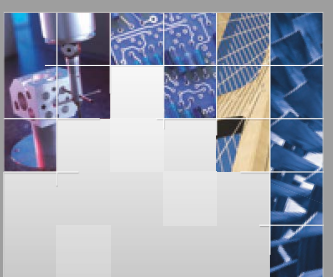

\section{Enfincering}
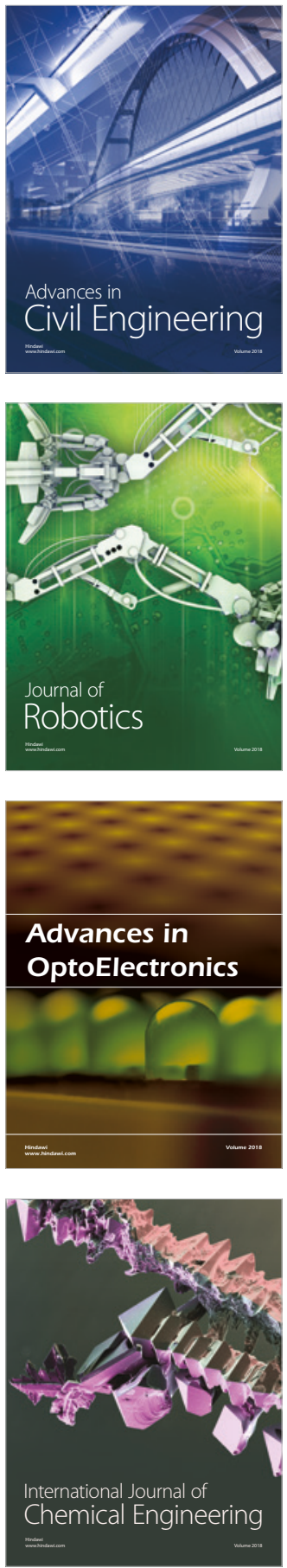

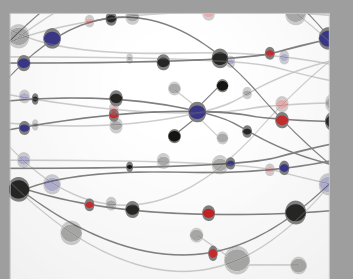

\section{Rotating \\ Machinery}

The Scientific World Journal

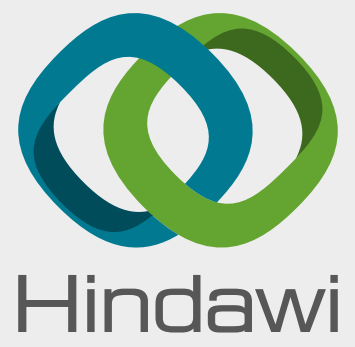

Submit your manuscripts at

www.hindawi.com
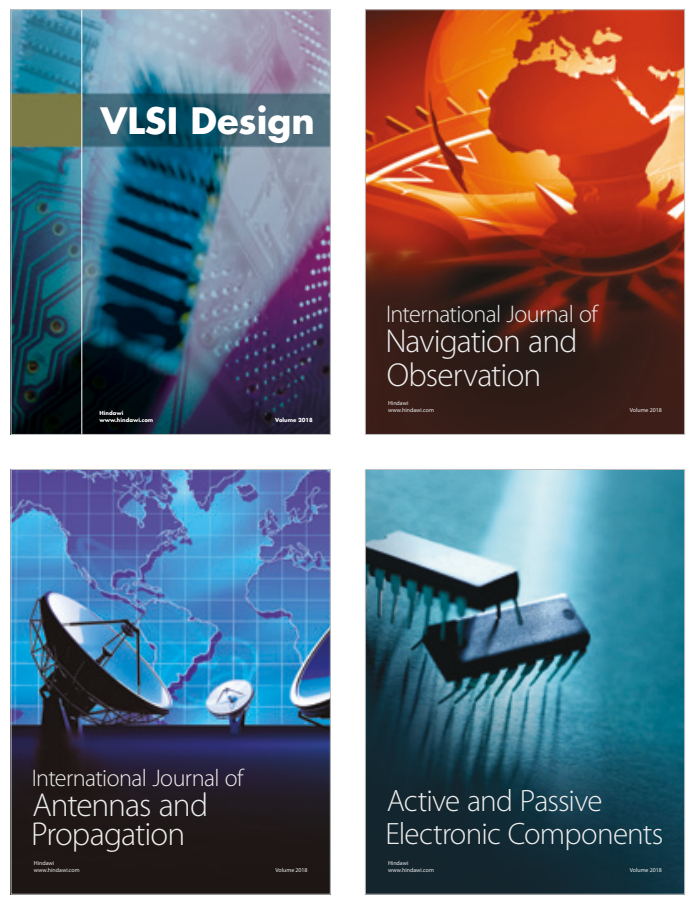
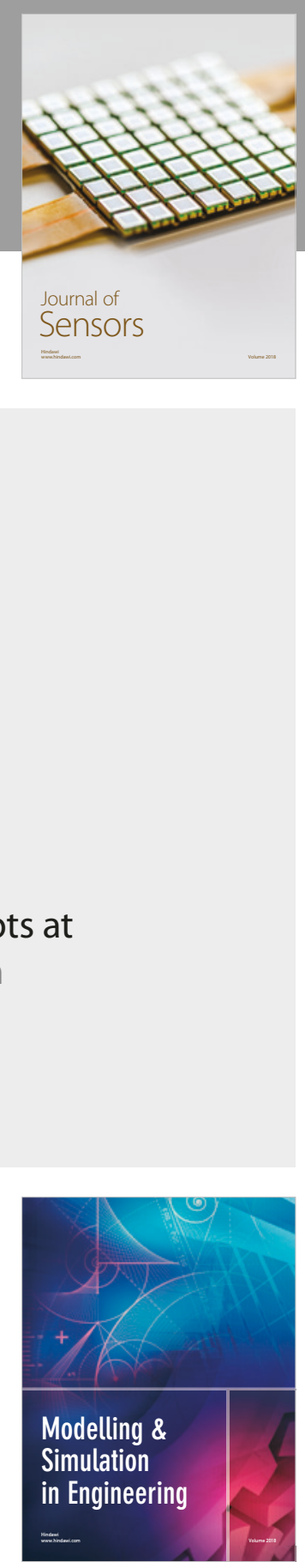

\section{Advances \\ Multimedia}
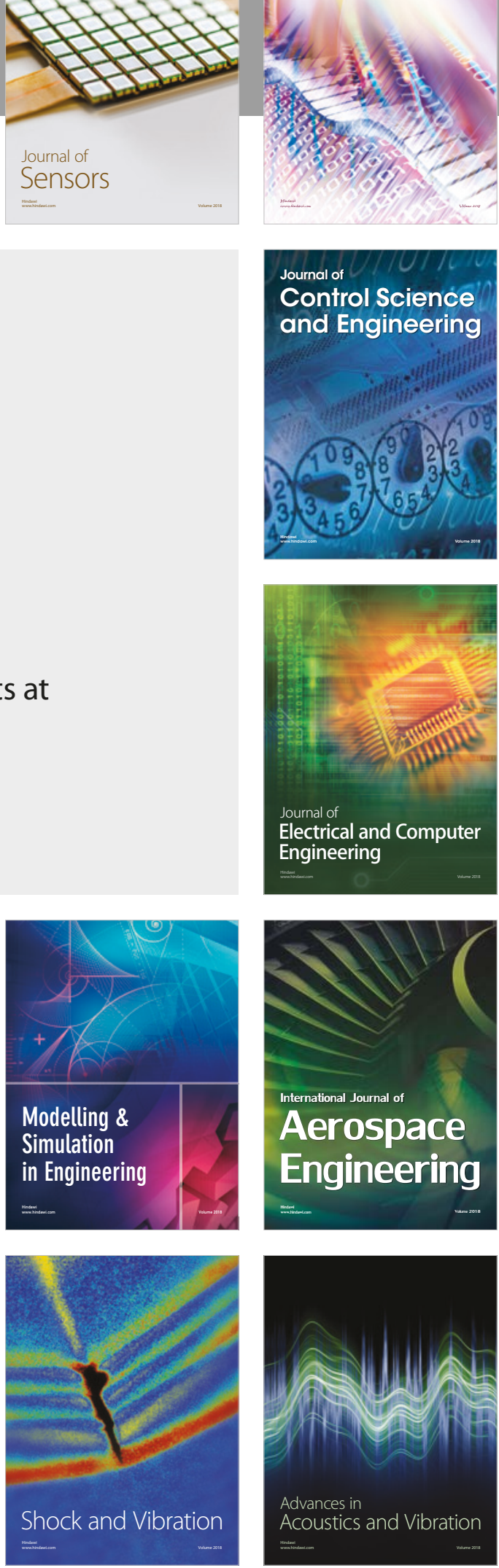